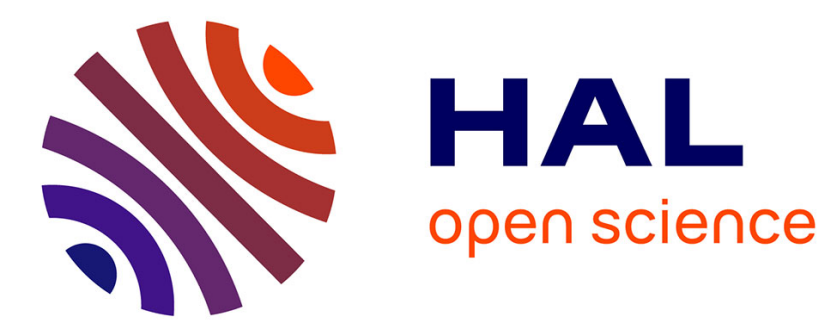

\title{
Robust Stabilization of Competing Species in the Chemostat
}

Alex dos Reis de Souza, Denis Efimov, Andrey Polyakov, Jean-Luc Gouzé

\section{To cite this version:}

Alex dos Reis de Souza, Denis Efimov, Andrey Polyakov, Jean-Luc Gouzé. Robust Stabilization of Competing Species in the Chemostat. Journal of Process Control, 2020, 87, pp.138-146. 10.1016/j.jprocont.2020.01.010 . hal-02462162

\section{HAL Id: hal-02462162 \\ https://hal.inria.fr/hal-02462162}

Submitted on 31 Jan 2020

HAL is a multi-disciplinary open access archive for the deposit and dissemination of scientific research documents, whether they are published or not. The documents may come from teaching and research institutions in France or abroad, or from public or private research centers.
L'archive ouverte pluridisciplinaire HAL, est destinée au dépôt et à la diffusion de documents scientifiques de niveau recherche, publiés ou non, émanant des établissements d'enseignement et de recherche français ou étrangers, des laboratoires publics ou privés.

\section{(ㅇ)(1) $\$$}

Distributed under a Creative Commons Attribution - NonCommercial - NoDerivatives| 4.0 


\title{
Robust Stabilization of Competing Species in the Chemostat *
}

\author{
Alex dos Reis de Souza ${ }^{a, *}$, Denis Efimov ${ }^{a}$, Andrey Polyakova ${ }^{\mathrm{a}}$, Jean-Luc Gouzéb \\ ${ }^{a}$ Inria - Lille Nord Europe, Université de Lille CNRS, UMR 9189 - CRIStAL, F-59000 \\ Lille, France \\ ${ }^{b}$ Université Côte d'Azur, Inria, INRA, CNRS, Sorbonne Université, Biocore Team, Sophia \\ Antipolis, France.
}

\begin{abstract}
This work addresses the problem of robust stabilization of the concentrations of two different species of living organisms, which compete for a single limiting substrate in a bioreactor. This stabilization is performed using discontinuous feedback control laws that ensure the coexistence of all species. The control laws are designed considering bounded parametric uncertainties on the kinetic rates.
\end{abstract}

Keywords: Robust control, sliding-mode, chemostat, biological process

\section{Introduction}

In the modern applications of biotechnology and biochemistry, the bioreactor is a well-known device that allows experiments involving living microorganisms, to be performed under controlled conditions and that mimics a natural environment. Many experiments and applications are conducted in such a device, like the production of pharmaceutical products and biofuels (such as methane and ethanol), yeast fermentation, polymerization, cultivation of cells and others. Practically, a bioreactor has three distinct modes of operation [1: batch, fed-batch or continuous. In the first, media is added and the process allowed to proceed until a certain condition is reached, in the second the fresh media can be continuously fed into the bioreactor but not removed and, finally, the third comprehends the case in which fresh media might be added and removed proportionally. A well-known example of a continuous bioreactor is the chemostat.

The problem of stabilization of the chemostat was widely studied over the last decades and many approaches for designing control laws with such an objective have been discussed. In the literature, there are many interesting

\footnotetext{
^This work was supported by IPL COSY.

* Corresponding author

Email address: alex.dos-reis-de-souza@inria.fr (Alex dos Reis de Souza)
} 
works addressing feedback control [2] 3], adaptive control [4] and robust control [5] 6] 7 , 8.

Indeed, robustness is an important feature when dealing with control systems, since real and practical applications are often plagued by uncertainties, exogenous disturbances or even unmodeled dynamics. In this sense, a control law is said to be robust if it guarantees stability (and performance up to some limitations) in spite of such inherent errors. In bioprocesses, the main source of uncertainty relates to the description of biological mechanisms $[9]$.

The competitive exclusion principle [10] states that the competition between $N$ species for a single limiting substrate results in the extinction (or wash-out) of all but one species, i.e., the one which has the best affinity with the limiting substrate. This fact encourages the development of methodologies to overcome such a drawback in laboratory-like environments, since coexistence would allow, for instance, deeper studies on the biological mechanisms of each species, their interaction and also their behavior in a community. The latter topic is very interesting since synthetic microbial consortia (see 11]) has shown that the interaction of different species might promote an improvement of productivity indexes. Indeed, the rise of applications consisting of more complex microbial communities is an interesting challenge for control theory and new methodologies are envisaged.

In this work, we present a novel result on the stabilization of a continuous bioreactor, consisting of two species that compete for a single limiting nutrient. We consider that the kinetic rates, which describe nutrient uptake by each species, are uncertain (and possibly time-varying) concerning their parameters. In this sense, we study the applicability of discontinuous control laws - more specifically, sliding-mode control - that guarantee persistence of both species, despite these uncertainties.

Sliding-mode control is a discontinuous and non-linear control method, being widely known for characteristics such as simplicity of design, finite-time rates of convergence, compensation of matched disturbances and robustness with respect to measurement noise and delays [12. This technique has proven its usefulness to a wide range of applications, such as regulation, trajectory tracking and, observation.

To the best of our knowledge, the current literature mainly focuses on the stabilization of bioreactors containing a single species and/or in a non-competitive environment (which is classically the case of anaerobic digestion), and some results on robustness have been attained (see, for instance, 3 3] 13] 14]). Then, the novelty of our methodology resides on the fact that we aim robust stabilization, at certain set-points, of the concentration of each competing species. Also, although not often explored in the literature, we show that the use of the substrate inflow concentration ( $S_{i n}$ in the following) as a controlled input is advantageous since it allows the use of an extra degree of freedom for the regulation of the species.

Furthermore, the methodology we developed aims to simplify and to drop harsh assumptions that might impose difficulties for future practical implementations. These assumptions often rely on the form of the kinetic function (many 
different functions, depicting different conditions and scenarios, have been reported in the literature [15]) or on the certainty/variability of its parameters.

Structure of the paper: the problem statement is presented in Section 2. Preliminary concepts are introduced in Section 3 and the main results are stated in Section 4. Then, Section 5 illustrates the inteded application through numerical examples and, finally, Section 6 gives concluding remarks and discusses future directions of this study.

This work is an extension of a previous conference paper [16, but this extended version presents substantial improvements.

\section{Problem Statement}

Consider the following non-linear system, which describes bacterial growth of 2 distinct species inside a chemostat with a single limiting substrate (timedependence was omitted for readability):

$$
\begin{aligned}
\frac{d S}{d t} & =\left(S_{i n}-S\right) D-\sum_{i=1}^{2} \rho_{i} \mu_{i}(S) x_{i} \\
\frac{d x_{i}}{d t} & =\left(\mu_{i}(S)-D\right) x_{i} \quad i=1,2 \\
S(0) & >0 \\
x_{i}(0) & >0 \quad i=1,2
\end{aligned}
$$

where $S$ and $x_{i}$ are, respectively, the concentration of the substrate and the $i$-th species, $S_{\text {in }}$ and $D$ are the control inputs (nutrient inflow concentration and dilution rate, respectively), which are supposed to be limited such as $S_{i n} \in$ $\left[0, S_{i n}^{\max }\right]$ and $D \in\left[0, D_{\max }\right]$. For simplicity, we will consider constant yield rates $\rho_{i}$ and the kinetic functions $\mu_{i}(S)$ - the specific growth rate - to be given by Monod's law, as follows:

$$
\mu_{i}(S)=\frac{a_{i} S}{b_{i}+S}
$$

where $a_{i}$ and $b_{i}$ are supposed to be uncertain, such that $a_{i} \in A_{i}=\left[a_{i}, \overline{a_{i}}\right]$ and $b_{i} \in B_{i}=\left[\underline{b_{i}}, \overline{b_{i}}\right]$ for $i=1,2$, where $\underline{a_{i}}, \overline{a_{i}}, \underline{b_{i}}, \overline{b_{i}}$ are known bounds, obtained by experiments and identification. These uncertainties are related to fluctuations that might happen in the microbial population, due to internal biological mechanisms or environmental influence (such as temperature or $\mathrm{pH}$, for instance). Furthermore, we will consider that these uncertain parameters might be timevarying (although respecting the aforementioned bounds).

For brevity in the following, let us define the general lower and upper bounds on the function $\mu_{i}(S)$ as follows:

$$
\begin{aligned}
& \underline{\mu_{i}}(S)=\min _{\substack{a_{i} \in A_{i} \\
b_{i} \in B_{i}}} \mu(S)=\frac{\underline{a_{i} S}}{\overline{b_{i}}+S} \\
& \overline{\mu_{i}}(S)=\max _{\substack{a_{i} \in A_{i} \\
b_{i} \in B_{i}}} \mu(S)=\frac{\overline{a_{i}} S}{\underline{b_{i}}+S}
\end{aligned}
$$


furthermore, as we will see, other types of kinetic functions can also be addressed by our approach.

The aim of this paper is then to design a robust control methodology, that ensures a solution of the stabilization problem (given below), despite the aforementioned uncertainties and stabilizes each one in a set-point.

In this sense, as it will be a key point in this development, let us introduce the following assumption:

Assumption 1. Defining $\xi(S)=\mu_{1}(S)-\mu_{2}(S)$, for any $a_{i} \in A_{i}$ and $b_{i} \in B_{i}$, let there exist a constant $S_{a, b}$ such that $\xi\left(S_{a, b}\right)=0$ and $\xi(S)>0$ for $S>S_{a, b}$.

This hypothesis states that the kinetic rates intersect, which is an obligatory requirement to have co-existence of the species (see [2] for an extensive discussion), while the latter property can be guaranteed by a proper numbering of each species.

Assumption 2. For any $a_{i} \in A_{i}$ and $b_{i} \in B_{i}$, the equality $\frac{\partial \xi\left(S_{o}\right)}{\partial S}=0$ is satisfied for some $S_{o}<S_{a, b}$.

This hypothesis just implies that the function $\xi(S)$ and its derivative with respect to $S$ are not zero at the same point. As it will be shown in the design of the control architecture, the distinction of these points is needed to achieve the stabilization of $x_{2}(t)$.

Remark 1. Assumptions 12 are fulfilled, for instance, with a sufficient (but not necessary) condition $\underline{a_{1}}>\overline{a_{2}}$ and $\overline{b_{1}}<\underline{b_{2}}$.

Semi-stabilization problem: Design feedback control laws $S_{\text {in }}: \mathbb{R}^{3} \rightarrow \mathbb{R}$ and $D: \mathbb{R}^{3} \rightarrow \mathbb{R}$ such that system (1) has its state vector $\chi:=\left(x_{1}, x_{2}\right)$ permanent (i.e., assuring coexistence), while stabilizing $x_{1}$ at desired concentration levels $x_{1}^{d}$ and $x_{2}$ at a certain point.

Furthermore, in this initial work, we suppose that measurements of the whole state vector are available.

\section{Preliminaries}

\subsection{Preliminaries on the Chemostat model}

The chemostat model, as given by (1), is well studied in the literature of both biotechnology and control systems. In the latter, early works such as [17] 18, 19] explored conditions in which permanence (or coexistence) is attainable in a competitive environment.

Indeed, it is well known that the coexistence of two species in the chemostat is possible if the nutrient uptake functions $\mu_{1}(S)$ and $\mu_{2}(S)$ intersect. In this light, if the dilution rate $D$ and the nutrient influx $S_{i n}$ are constant, the following scenarios are possible [19] (the reader is invited to refer to proofs therein) with equilibrium points $\Omega_{i}=\left\{\left(x_{1}^{e q}, x_{2}^{e q}\right) \in \mathbb{R}_{+}^{2}\right\}$ :

1. Substrate depletion: $S=0$ and $\Omega_{1} \in \mathbb{R}_{+}^{2}$ (if $D=0$ );

2. Washout: $S=S_{\text {in }}$ and $\Omega_{2}=(0,0)$; 
3. Mono-species: $S=S^{*}$ and $\Omega_{3}=\left(x_{1}^{*}, 0\right)$;

4. Coexistence: $S=S^{*}$ and $\Omega_{4}=\left(x_{1}^{d}, x_{2}^{d}\right)$;

where $x_{i}^{d}$ are specified concentrations and $x_{1}^{*}=\left(S_{i n}-\frac{b_{1}}{\frac{a_{1}}{D}-1}\right)$.

Considering the possible equilibria above, the first case relates to the case where $D=0$, causing the initial substrate concentration to be consumed until $S=0$. The second situation relies on the case where the dilution rate is greater than the maximum growth rate of the $i^{\text {th }}$ species. The third relates to the case where only one species survives (hence the competition exclusion holds) and this point is asymptotically stable [19]. Finally, the fourth case relates to the solution of the permanence problem, where all species coexist.

Washout is never an interesting scenario, since all living biomass would be eradicated. The mono-species case also is not interesting, since one might aim to obtain some benefit from the interaction between both species. The coexistence is hence an interesting option and design control laws that allow pertinent behavior on $\Omega_{4}$ (such as set-point stabilization or maximization) is desirable.

\subsection{Preliminaries on Sliding Mode Control}

Here, we present a brief introduction to sliding-mode control and the design methods used in the paper. Consider the following non-linear system:

$$
\dot{x}(t)=f(t, x, u, d)
$$

where $x \in \mathbb{R}^{n}$ is the state vector, $u \in \mathbb{R}^{m}$ is the control input vector and $d \in \mathbb{R}^{n}$ is a vector containing perturbations and possibly uncertainties within the system. Let us start considering a first-order conventional sliding mode. Consider the following surface in the state space:

$$
\mathbb{S}=\left\{x \in \mathbb{R}^{n}, \sigma(x)=0\right\}
$$

where $\sigma: \mathbb{R}^{n} \rightarrow \mathbb{R}$ is a continuously differentiable function called sliding variable.

The intention is to design $\sigma(x)$ in such a way that system (4), under feedback control, behaves with prescribed performance. In this sense, if the control input is selected guaranteeing $\dot{\sigma}=-\eta \operatorname{sign}(\sigma)$, where $\eta>0$, one can consider a Lyapunov function as

$$
V=\frac{1}{2} \sigma^{2}
$$

and, computing its time derivative, we have that

$$
\dot{V}=\sigma \dot{\sigma}=-\eta|\sigma|
$$

which implies $\dot{V} \leq 0$, hence the origin of $\sigma(x)$ is globally stable. Moreover, it is clear that $|\sigma|=\sqrt{2} V^{0.5}$ and, consequently, it implies that

$$
V^{0.5}(t)=\max \left\{0, V^{0.5}(0)-\sqrt{2} \eta t\right\}
$$

meaning that the solution of (6) becomes zero in a finite time [20]. 
Here, we can discriminate two interesting phases of this methodology: the reaching phase, which is guaranteed if condition (6) is satisfied, it describes the motion of the system towards the surface $\sigma(x)$, and the sliding phase, describing the motion of the system in the surface $\sigma(x)=0$.

Recently, the concept of higher-order sliding-mode control has been widely studied. The techniques developed in this framework extend all interesting properties of standard sliding-mode control to systems with a relative degree greater than one. To introduce this concept, consider the case of relative degree 2 and assume that the dynamics of the sliding variable $\sigma$ satisfies the following non-linear system:

$$
\ddot{\sigma}=a(t, x(\cdot))+b(t, x(\cdot)) u(t, \sigma, \dot{\sigma})
$$

where $|a(t, x(\cdot))| \leq C$ and $0<b_{\min } \leq b(t, x(\cdot)) \leq b_{\max }$, for all $x \in \mathbb{R}$. Also, constants $C \geq 0$ and $0<b_{\min } \leq b_{\max }$ are supposed to be known. Many controllers have been proposed in order to steer $\sigma$ and $\dot{\sigma}$ to zero in finite time, such as the twisting controller [21, the suboptimal controller [22, quasi-continuous controllers [23] and others.

As it will be used on the development of this work, we will further discuss the properties of the suboptimal controller. This algorithm is given by the control law 22 .

$$
u=-k_{1} \operatorname{sign}[\Delta(t)]
$$

where $k_{1}, \lambda>0$ and

$$
\Delta(t)=\Delta(t, \sigma(\cdot)):=\sigma(t)-\lambda \sigma\left(t^{*}\right)
$$

where $t^{*}$ is the last instant of time in which $\dot{\sigma}\left(t^{*}\right)=0$, i.e.,

$$
t^{*}=t^{*}(t, \dot{\sigma}(\cdot)):=\sup _{\tau \leq t: \dot{\sigma}(\tau)=0} \tau
$$

As one can see, control law (8) is a functional, since it requires information of its current and past trajectories of the system states. Also, it is interesting to notice that this control law does not require information on $\dot{\sigma}(t)$, but only the detection of an event where $\dot{\sigma}(t)=0$. This clearly offers an advantage for practical implementations, since information of $\dot{\sigma}(t)$ might not be always available and its estimation might suffer from numerical complications.

Also, invoking the work presented in [24, a candidate Lyapunov function for such a controller is given by

$$
\begin{aligned}
V_{\text {sub }}(t, x(\cdot), \sigma(\cdot))= & p \sqrt{\left|x(t)-\frac{\sigma^{2}(t)}{2\left(-C \operatorname{sign}(\Delta(t))-b_{\max } k_{1} \operatorname{sign}(\Delta(t))\right)}\right|} \\
& -\frac{\sigma(t)}{-C \operatorname{sign}(\Delta(t))-b_{\max } k_{1} \operatorname{sign}(\Delta(t))}
\end{aligned}
$$

where 
- $0<\lambda<1, \quad b_{\min } k_{1}>C$ and

$$
\frac{\lambda}{1-\lambda}>\frac{1}{k}+k-1, \quad k:=\frac{b_{\max } k_{1}+C}{b_{\min } k_{1}-C}
$$

- And, finally,

$$
p:=2 \sqrt{\frac{1}{b_{\max } k_{1}+C}\left(\frac{\lambda}{1-\lambda} k^{2}+\sqrt{\left(\frac{\lambda}{1-\lambda}\right)^{2} k^{4}-1}\right)} ;
$$

and, additionally, an estimate of the reaching time is given by

$$
t_{\text {reach }} \leq t^{\prime}+k p \sqrt{\left|\sigma\left(t^{\prime}\right)\right|}
$$

where $t^{\prime}$ is the first moment in time such that $\dot{\sigma}(t)=0$.

Corollary 3.1. Consider the suboptimal control (8) and dynamics (7). Let $\sigma(0)=0$ and $\dot{\sigma}(0)>0$, then the following estimates are satisfied for all $t \geq 0$ :

$$
\begin{aligned}
& |\dot{\sigma}(t)| \leq|\dot{\sigma}(0)| \\
& |\sigma(t)| \leq\left|\sigma\left(t^{\prime}\right)\right|
\end{aligned}
$$

where $t^{\prime}=\{\inf t \geq 0: \dot{\sigma}(t)=0\}$.

Proof. This proof relies on the integration of the expression of the worst-case trajectories for the closed-loop system, i.e., $\ddot{\sigma}(t) \leq C-b_{\min } k_{1} \operatorname{sign}(\Delta(t))$. Initially, as $\dot{\sigma}(0)>0$ and $\sigma(0)=0$, we have that $\ddot{\sigma} \leq-r$, for $r=b_{\min } k_{1}-C>0$.

Considering a general time interval $\left[t_{0}, t\right]$ (where $t_{0}$ is a certain initial time instant), the integration of $\ddot{\sigma}(t)$ results in

$$
\begin{aligned}
& \dot{\sigma}(t) \leq \dot{\sigma}\left(t_{0}\right)-r\left(t-t_{0}\right) \\
& \sigma(t) \leq \sigma\left(t_{0}\right)+\dot{\sigma}\left(t_{0}\right)\left(t-t_{0}\right)-r \frac{\left(t-t_{0}\right)^{2}}{2}
\end{aligned}
$$

Evaluating (14) in the time interval $\left[0, t^{\prime}\right]$, we can conclude that

$$
t^{\prime} \leq \frac{\dot{\sigma}(0)}{r}
$$

and

$$
\sigma\left(t^{\prime}\right) \leq \frac{\dot{\sigma}^{2}(0)}{2 r}
$$

Then, it is clear that the first sign change in the control law will take place in a second instant of time $t^{\prime \prime}>t^{\prime}$, in which we have (due to the form of (8)) 
that $\sigma\left(t^{\prime \prime}\right)=\lambda \sigma\left(t^{\prime}\right)$. Consequently, the evaluation of (14) in the time interval $\left[t^{\prime}, t^{\prime \prime}\right]$ shows that

$$
t^{\prime \prime} \leq \frac{\dot{\sigma}^{2}(0)}{r}(\sqrt{1-\lambda}+1)
$$

and hence, we can finally conclude that

$$
\left|\dot{\sigma}\left(t^{\prime \prime}\right)\right| \leq-|\dot{\sigma}(0)| \sqrt{1-\lambda}
$$

As $\sqrt{1-\lambda}<1$ for $0<\lambda<1$, it is clear that $\left|\dot{\sigma}\left(t^{\prime \prime}\right)\right|<|\dot{\sigma}(0)|$. Hence, as contraction and convergence properties of such a control algorithm have been proven (see [22] and proofs therein), we can then conclude that $|\dot{\sigma}(t)| \leq|\dot{\sigma}(0)|$ for all $t>0$.

The proof of the remaining estimate follows by analysing the signal change of the control law. Clearly, due to the functioning of the functional $\Delta(\sigma(t))$, the signal of the control law will switch every time that $\dot{\sigma}(t)$ reaches zero or when $\sigma(t)=\lambda \sigma\left(t^{*}\right)$ (see the definition of $t^{*}$ above). This fact allows us to deduce that, as $\dot{\sigma}\left(t^{\prime \prime}\right)<0$, it is clear that $\dot{\sigma}(t)<0$ holds for all $t>t^{\prime}$. Thus $|\sigma(t)|<\left|\sigma\left(t^{\prime}\right)\right|$ holds for all $t>0$, as claimed.

\section{Design of the Control Architecture}

Our control strategy has two phases. First, using the control $S_{\text {in }}$ we are going to shift the substrate concentration to a positive value ensuring the system controllability, and in parallel to stabilize $x_{1}$ at a desired position $x_{1}^{d}>0$ applying the control $D$. Once these goals are reached, we will commute the control goal for $S_{i n}$ to the stabilization of $x_{2}$ at a constant level. Below all these control laws are described in details and their properties are substantiated.

The commutation between control phases is performed by a supervisor scheme, whose logic is given by:

- Stabilization of $x_{1}$ is always activated;

- The first switching law is to be activated for $t<t_{\text {switch }}$;

- The second switching law is to be activated for $t \geq t_{\text {switch }}$;

where $t_{\text {switch }}$ is the first instant of time in which $\left|x_{1}-x_{1}^{d}\right|=0$.

\subsection{Stabilization of $x_{1}$}

Consider the following decision variable:

$$
\sigma_{1}\left(x_{1}\right)=x_{1}-x_{1}^{d}
$$

where $x_{1}^{d}>0$ is an arbitrary point for $x_{1}$ to be stabilized.

As we can see from equation (17), if the concentration $x_{i}$ is in a steady-state position, i.e., $\dot{x}_{i}(t)=0$ for $t \geq 0$, then there are two possibilities: either $x_{i}=0$ or $D(t)=\mu_{i}(S(t))$. With the latter case in mind, we can state the following result: 


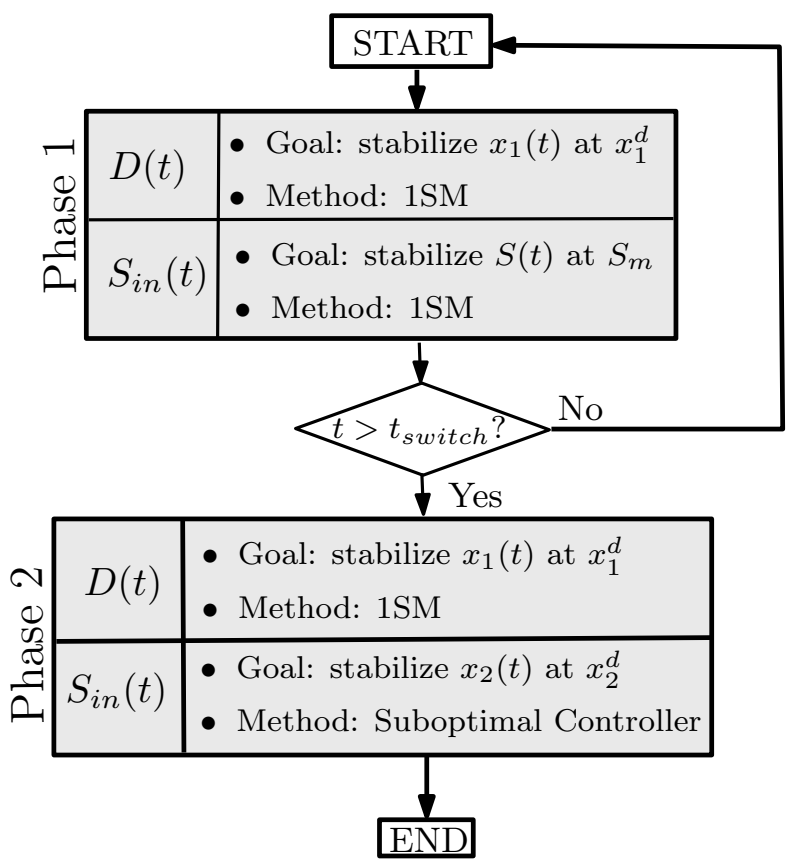

Figure 1: Overview of the control architecture

Proposition 4.1. Consider model (1) and surface (15). If the dilution rate is selected such as

$$
D= \begin{cases}\overline{\mu_{1}}(S)+\chi, & \text { if } \sigma_{1} \geq 0 \\ \underline{\mu_{1}}(S)-\chi, & \text { if } \sigma_{1}<0\end{cases}
$$

where $\chi \in\left(0, \mu_{1}(S(0))\right)$ is a tuning parameter and, considering the aforementioned bounds on $\mu_{i}(S)$, then the closed-loop system is globally finite-time stable with respect to the output $\sigma_{1}$, provided that $S(t) \geq S(0) \quad \forall t \geq 0$.

Proof. Consider the following Lyapunov function candidate for (15), which is proper and positive definite with respect to $\sigma_{1}$ :

$$
V_{1}=\frac{1}{2} \sigma_{1}^{2}
$$

whose time derivative is given by $\dot{V}_{1}=\sigma_{1} \dot{\sigma}_{1}$. By noticing that $\sigma_{1}=\sqrt{2 V_{1}}$, we can rewrite it so $D$ appears explicitly:

$$
\dot{V}_{1}=\sigma_{1}\left(\mu_{1}(S)-D\right) x_{1}
$$

and hence, selecting $D$ as given by $(16)$ and stressing that $x_{1}(0)$ can be either lower or greater than $x_{1}^{d}$, we can conclude that $\dot{V}_{1} \leq-\chi \sqrt{2 V_{1}} \min \left\{x_{1}(0), x_{1}^{d}\right\}$ holds, proving the claim. 
An immediate consequence of this choice on $D$ is the fact that, when $x_{1}$ is stabilized at level $x_{1}^{d}$, we will have an equivalent control $D_{e q}=\mu_{1}(S)$.

Clearly, the given control law for $D$ uses information about $x_{1}$ and also $S$, and the latter has to be separated from zero (bigger than $S(0)>0$ to have $D(t)>0$ for the selected value of $\chi)$. Hence, it is necessary to design a control law for $S$, which can be done using $S_{i n}$.

\subsection{First Control Law $-S_{i n}^{[1]}$}

The first control strategy is to be active whenever $x_{1}$ is not stabilized in surface (15) or its vicinity. The objective here is to keep $S$ away from zero, otherwise it would cause $D=0$ (due to $\mu_{1}(0)=\mu_{2}(0)=0$ ) and we will have no control action on $x_{1}$. Moreover, it is also necessary to realize the condition imposed in Theorem 4.1 that $S(t) \geq S(0)$ for all $t \geq 0$.

In order to design a control law for $S_{i n}$, it is necessary to note that the point $S_{o}$, defined in Assumption 2, corresponds to the value of $S$ at which the system loses its controllability for the variable $x_{2}$ with respect to the control input $S_{\text {in }}$ (see below the analysis of control for the second phase: at $S_{o}$ the dynamics of variables $x_{2}$ and $\dot{x}_{2}$ is independent in $S_{i n}$, hence, the system is not controllable). In addition, the system is not controllable at all if $S=0$. Then the goal is to shift $S$ out of the dangerous region (see figure 2 below).

To this end, let us introduce three special points: $S_{a, b}, \overline{S_{o}}$ and $S_{m}$, which are given by

$$
\begin{aligned}
\overline{S_{o}} & =\max _{\substack{a_{i} \in A_{i} \\
b_{i} \in B_{i}}} S_{o, i} \\
\underline{S_{a, b}} & =\min _{\substack{a_{i} \in A_{i} \\
b_{i} \in B_{i}}} S_{a_{i}, b_{i}}
\end{aligned}
$$

hence, $S_{m}$ is selected such as $S_{m}>\overline{S_{0}}$.

Remark 2. An option for selection of $S_{m}$ is $S_{m}=\frac{\overline{S_{o}}+S_{a, b}}{2}$.

Thus stabilization of $S$ at $S_{m}$ will ensure that, at least at the instant of commutation to regulate $x_{2}$, the latter variable is controllable, avoiding the issue remarked above. Figure 2 illustrates the selection of such a zone, taking the uncertainties into account.

Hence, in this light, we can state the following result:

Proposition 4.2. Consider model (1). By means of a switching law given by

$$
S_{i n}^{[1]}=\frac{1}{D} \begin{cases}S_{\max }, & \text { if } S<S_{m} \\ 0, & \text { if } S \geq S_{m}\end{cases}
$$

where

$$
S_{\max }=D S+\sum_{i=1}^{2} \rho_{i} x_{i} \overline{\mu_{i}}(S)+\epsilon
$$




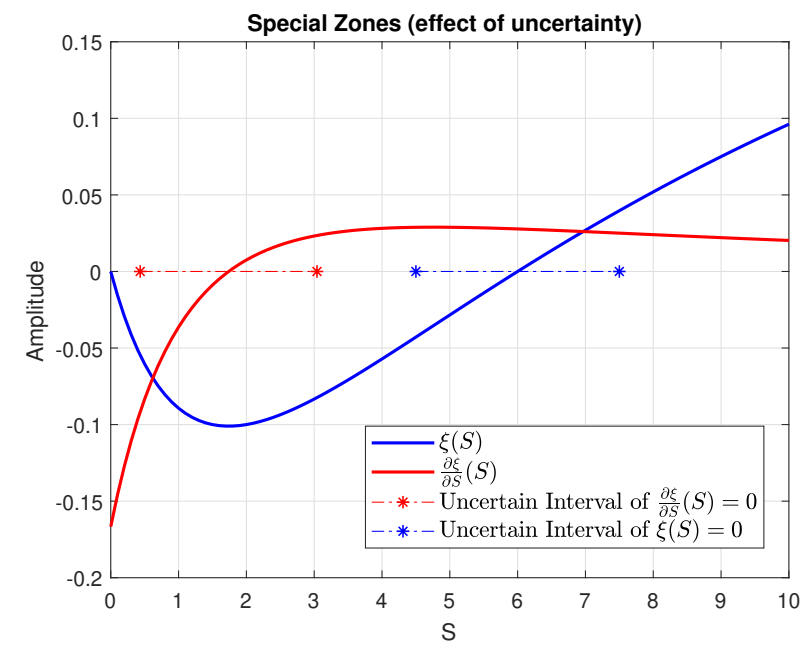

Figure 2: Illustration of the special zones in functions $\xi(S)$ and $\frac{\partial \xi}{\partial S}$. Controllability is lost when $\frac{\partial \xi}{\partial S}=0$, which is affected by the considered uncertainties.

with $\epsilon>0$, the output $S-S_{m}$ is globally finite-time stabilized for (1), provided that $D(t)>0$, for all $t \geq 0$.

Proof. : Consider the following candidate Lyapunov function:

$$
V_{S}(S)=\frac{1}{2}\left(S-S_{m}\right)^{2}
$$

which has time derivative given by

$$
\dot{V}_{S}=\left(S-S_{m}\right) \dot{S}=\left(S-S_{m}\right)\left(D\left(S_{i n}^{[1]}-S\right)-\sum_{i=1}^{2} \rho_{i} x_{i} \mu_{i}(S)\right)
$$

and note that with the selected commutation law, one has $\dot{V}_{S} \leq-\varepsilon \sqrt{2 V_{s}}$ for some $\varepsilon \in(0, \epsilon]$ dependent on initial conditions, implying the stated result.

Theorem 4.3. Let $S(0) \leq S_{m}$. Then controls (16), (18) with $\chi \in\left(0, \mu_{1}(S(0))\right)$ and $\epsilon>0$ ensure that $x_{1}(t)=x_{1}^{d}$ and $S(t)=S_{m}$ for all $t \geq T$, where $T>0$ is a finite time dependent on the initial conditions.

Proof. First, note that such a selection of $\chi$ induces $D(t)>0$. Furthermore, as proven in Proposition 4.2 by means of Lyapunov function (17), the origin of (15) is globally finite-time stable after a time $T_{1}>0$ bounded by (see subsection $3.3)$

$$
T_{1} \leq \frac{V_{1}^{0.5}\left(x_{1}(0)\right)}{\min \left\{x_{1}(0), x_{1}^{d}\right\} \chi \sqrt{2}}
$$


Consequently, control law 18 is well-posed (since $D(t)>0$ guarantees that no division by zero will occur). If $\epsilon>0$, as proven in Proposition 2 by means of Lyapunov function (19), control law (18) renders the system with output $S-S_{m}$ globally finite-time stable after a time $T_{2}>0$ bounded by

$$
T_{2} \leq \frac{V_{S}^{0.5}(S(0))}{\varepsilon \sqrt{2}}
$$

Finally, control laws (16) and (18) render $x_{1}(t)=x_{1}^{d}$ and $S(t)=S_{m}$ after a time $T=T_{1}+T_{2} \leq \frac{1}{\sqrt{2}}\left(\min \left\{x_{1}(0), x_{1}^{d}\right\} \chi^{-1} V_{1}^{0.5}\left(x_{1}(0)\right)+\varepsilon^{-1} V_{S}^{0.5}(S(0))\right)$, as claimed.

\subsection{Second Control Law $-S_{\text {in }}^{[2]}$}

The second control has to be active once $x_{1}$ is stabilized on the surface (15). The objective here is then to stabilize the remaining species $x_{2}$ at an arbitrary point $x_{2}^{d}>0$, which can be done by means of $S_{i n}$.

Remark 3. As $x_{1}$ is in sliding motion due to the discontinuous control laws developed previously, an immediate consequence is that after the establishment of the sliding motion for the variable $\sigma_{1}$, the control $D$ can be considered in its equivalent form 25] $D_{e q}=\mu_{1}(S)$, which is assumed in this subsection.

As it can be seen from model (1), if concentration $x_{2}$ is in steady-state, i.e., $\dot{x}_{2}=0$, the equivalent control on $D$ implies that stabilization can be achieved if $S$ is steered to a certain level in which $\xi(S)=0$ holds, i.e., $S=S_{a_{i}, b_{i}}$. Indeed, if all parameters of the kinetic rates (2) were perfectly known, then the intersection point $S_{a_{i}, b_{i}}$ would be readily available and the stabilization is easily solved. However, as aforementioned, these parameters are uncertain and therefore the control law designed in this section must provide robust stabilization of $x_{2}$.

Before stating the main results of this subsection, let us define the bounds on $\xi$ (in terms of (3)) as follows:

$$
\begin{aligned}
& \bar{\xi}(S)=\overline{\mu_{1}}(S)-\underline{\mu_{2}}(S) \\
& \underline{\xi}(S)=\underline{\mu_{1}}(S)-\overline{\overline{\mu_{2}}}(S)
\end{aligned}
$$

also, the bounds on the derivatives of $\xi$ with respect to $S$ are given by

$$
\begin{aligned}
& \frac{\partial \bar{\xi}}{\partial S}=\frac{\overline{a_{1}} \overline{b_{1}}}{\left(\underline{b_{1}}+S\right)^{2}}-\frac{\underline{a_{2} b_{2}}}{\left(\overline{b_{2}}+S\right)^{2}} \\
& \frac{\partial \underline{\xi}}{\partial S}=\frac{\underline{a_{1} b_{1}}}{\left(\overline{b_{1}}+S\right)^{2}}-\frac{\overline{a_{2}} \overline{b_{2}}}{\left(\underline{b_{2}}+S\right)^{2}}
\end{aligned}
$$

In this sense, let us define a decision variable $y=x_{2}-x_{2}^{d}$ and, taking Remark 3 into account, we have that $\dot{y}=-\xi(S) x_{2}$. Differentiating this last expression with respect to time once more, one has that

$$
\ddot{y}=x_{2}\left(\xi^{2}(S)-\frac{\partial \xi}{\partial S}\left(D\left(S_{\text {in }}-S\right)-\sum_{i=1}^{2} \rho_{i} x_{i} \mu_{i}(S)\right)\right)
$$


where the control input $S_{i n}$ appears explicitly. Following the idea of the suboptimal control, this expression leads to the choice of control input $S_{i n}^{[2]}$ to be given by

$$
S_{i n}^{[2]}=S+\frac{1}{D}\left(\sum_{i=1}^{2} \rho_{i} x_{i} \tilde{\mu}_{i}(S)-k_{1} \operatorname{sign}[\Delta(y(t))]\right)
$$

where $\tilde{\mu}_{i}(S)=\frac{\bar{\mu}_{i}(S)+\underline{\mu}_{i}(S)}{2}$ is a middle point estimate of the uncertain function $\mu_{i}(S)$ that appears in 20 .

Then, by plugging control law (21) in the dynamics $(20)$, we have that

$$
\begin{aligned}
\ddot{y} & =x_{2}\left(\xi^{2}(S)-\frac{\partial \xi}{\partial S}\left(\sum_{i=1}^{2} \rho_{i} x_{i}\left(\tilde{\mu}_{i}(S)-\mu_{i}(S)\right)-k_{1} \operatorname{sign}[\Delta(y(t))]\right)\right) \\
& =a(t)+b(t) k_{1} \operatorname{sign}[\Delta(y(t))]
\end{aligned}
$$

where the terms $a(t)$ and $b(t)$ are given by

$$
\begin{aligned}
& a(t)=x_{2}\left(\xi^{2}(S)-\frac{\partial \xi}{\partial S} \sum_{i=1}^{2} \rho_{i} x_{i}\left(\tilde{\mu}_{i}(S)-\mu_{i}(S)\right)\right) \\
& b(t)=x_{2} \frac{\partial \xi}{\partial S}
\end{aligned}
$$

and hence this last expression clearly has the same form as (7). Also, in order to eliminate the uncertain term $\mu_{i}(S)$, we can write the bounds of functions $a(t)$ and $b(t)$ in $(23)$ as

$$
\begin{aligned}
& |a(t)| \leq x_{2}\left(\bar{\xi}^{2}(S)+\frac{\partial \bar{\xi}}{\partial S} \sum_{i=1}^{2} \rho_{i} x_{i}\left(\frac{\bar{\mu}_{i}(S)-\underline{\mu}_{i}(S)}{2}\right)\right)=a_{\max }\left(x_{1}, x_{2}, S\right) \\
& b_{\min }\left(x_{2}, S\right)=x_{2} \frac{\partial \xi}{\partial S}(S) \leq b(t) \leq x_{2} \frac{\partial \bar{\xi}}{\partial S}(S)=b_{\max }\left(x_{2}, S\right)
\end{aligned}
$$

and it is worth to notice that $b_{\min }\left(x_{2}, S\right)>0$ while $S>\overline{S_{o}}$.

Proposition 4.4. The selection of control law (21) renders $S_{\text {in }}$ non-negative, for all $t \geq 0$, provided that $k_{1} \leq D S+\sum_{i=1}^{2} \rho_{i} x_{i} \tilde{\mu}_{i}(S)$.

Proof. The proof is straightforward by noticing that $k_{1}$ is the only tunable parameter in (21) and, to assure positiveness of $S_{i n}$, the term $k_{1} \operatorname{sign}[\Delta(y(t))]$ must not compensate all other (positive) terms. Since $\operatorname{sign}[\Delta(y(t))] \in[-1,1]$ it is obvious that the selection of $k_{1}$ such as

$$
k_{1} \leq D S+\sum_{i=1}^{2} \rho_{i} x_{i} \tilde{\mu}_{i}(S)
$$

renders $S_{\text {in }} \geq 0$ for all $t>0$, as claimed. 
At this point, we can easily see that as long as term $\frac{\partial \xi}{\partial S}$ appears in $(20)$, it implies that if $S$ reaches the interval $S \leq \overline{S_{o}}$, the stabilization of surface $\sqrt{20}$ is no longer possible (actually the system loses its controllability at $S_{o}$, but since this value is uncertain we have to keep $S>\overline{S_{o}}$ ). Clearly, control law (21) does not guarantee a priori that $S$ will not reach this interval.

In order to state conditions to overcome such a problem and also to ensure a proper selection of $k_{1}$, let us deeper study what happens in such a control phase. Assume that $k_{1}$ is properly tuned and that $t_{2}$ is the time instant in which this control phase is activated. Since the previous control phase has stabilized $S$ at a level $S_{m}>\overline{S_{o}}$, we have that $\dot{x}_{2}\left(t_{2}\right)>0$ and hence two different scenarios are possible:

1. If $x_{2}\left(t_{2}\right)>x_{2}^{d}$, then $S$ must increase in order to have $\dot{x}_{2}<0$;

2. If $x_{2}\left(t_{2}\right)<x_{2}^{d}$, then $S$ must decrease in order to have $\dot{x}_{2}>0$;

Obviously, the second case is troublesome since, depending on the selected gain, $S$ might reach the region in which the stabilization is compromised. Hence, in this initial work, we will assume that the stabilization of surface $(20)$ will be proceed for $x_{2}^{d}=x_{2}\left(t_{2}\right)$. With this assumption, the problem of having $S \leq \overline{S_{o}}$ may be alleviated.

In the following, we consider an interval $I=[\underline{S}, \bar{S}] \times x_{1}^{d} \times\left[x_{2}^{d}-\delta, x_{2}^{d}+\delta\right]$ for all $t \geq t_{2}$ and some $\overline{S_{o}}<\underline{S}<\bar{S}<+\infty$ and $\delta>0$. Due to functioning of the suboptimal control, the dynamics of the closed-loop system will be governed by the equation $\ddot{y}(t)=a(t)-b(t) k_{1}$. If the choice of $k_{1}$ imposes that $a(t)<b(t) k_{1}$, then $\ddot{y}(t)=-r$ gives the worst-case trajectory estimate. Since in this case $y\left(t_{2}\right)=0$ and $\dot{y}\left(t_{2}\right)>0$, let us define, for $\left(S(t), x_{1}(t) x_{2}(t)\right) \in I$, the minimum value of $\ddot{y}(t)$ as $r=a_{\max }\left(x_{1}, x_{2}, S\right)-b_{\min }\left(x_{2}, S\right) k_{1}$ for the time interval $\left[t_{2}, t_{3}\right]$, where $t_{3}>t_{2}$ is the instant of time that $\dot{y}\left(t_{3}\right)=0$. Omitting all arguments for the sake of readability, this estimate can be expressed as follows:

$$
\begin{aligned}
r= & -\left[\left(x_{2}^{d}+\delta\right)\left(\xi(\bar{S})^{2}+\xi_{\max }^{\prime}\left(x_{1}^{d} \rho_{1} \frac{\bar{\mu}_{1}(\bar{S})-\underline{\mu}_{1}(\underline{S})}{2}+\left(x_{2}^{d}+\delta\right) \rho_{2} \frac{\bar{\mu}_{2}(\bar{S})-\underline{\mu}_{2}(\underline{S})}{2}\right)\right)\right. \\
& \left.-\left(x_{2}^{d}-\delta\right) \xi_{\min }^{\prime} k_{1}\right]
\end{aligned}
$$

where $\xi_{\min }^{\prime}=\min _{I} \frac{\partial \xi}{\partial S}$ and $\xi_{\max }^{\prime}=\max _{I} \frac{\partial \bar{\xi}}{\partial S}$ (this notation will be also used in the following).

In this light, let us state the following result:

Lemma 4.5. If there exists a compact interval I in which the following inequalities hold:

$$
\begin{aligned}
& \text { (a) } \frac{1}{\xi_{\min }^{\prime}}\left(\bar{\xi}^{2}(\bar{S})+\xi_{\max }^{\prime} \sum_{i=1}^{2} \rho_{i} \bar{x}_{i} \frac{\bar{\mu}_{i}(\bar{S})-\underline{\mu}_{i}(\underline{S})}{2}\right)<D \underline{S}+\sum_{i=1}^{2} \rho_{i} \bar{x}_{i} \tilde{\mu}_{i}(\underline{S}), \\
& \text { (b) }|\xi(S)| \leq \frac{\left|x_{2}^{d} \xi\left(S_{m}\right)\right|}{x_{2}^{d}-\delta} \text { and }
\end{aligned}
$$


(c) $\frac{1}{2 r}\left(x_{2}^{d} \xi\left(S_{m}\right)\right)^{2} \leq \delta$

for all $\left(S(t), x_{1}(t), x_{2}(t)\right) \in I, \overline{x_{1}}=x_{1}^{d}$ and $\overline{x_{2}}=x_{2}^{d}+\delta$, then there is a choice of constant gain $k_{1}$ in (21) that assures both positiveness of $S_{\text {in }}$ and the permanence of trajectories of $S$ and $x_{2}$ inside the domain of attraction of the origin of (20).

Proof. The idea here is to show that, under the conditions presented in this lemma, it is possible to select a constant value of $k_{1}$ that satisfies all constraints of non-negativeness and stabilizability of (20), during all transients.

First, the right-hand side of inequality (a) is immediate from Proposition 4.4, corresponding directly to the choice of $k_{1}$ that yields $S_{i n}$ non-negative. The left-hand side, however, relates to the choice of $k_{1}$ that stabilizes these dynamics. Indeed, one can easily see that control law (21) renders the dynamics (20) as

$$
\ddot{y}=a(t)+b(t) k_{1} \operatorname{sign}[\Delta(y(t))]
$$

where $a(t)$ and $b(t)$ are given by (23). Noticing that this last expression has the same form as (7), we know that the stabilization by the suboptimal control is possible if $b_{\min } k_{1}>\left|a_{\max }\right|$, hence

$$
k_{1}>\frac{\left|a_{\max }\right|}{b_{\min }}
$$

which, recalling (24) and the constraint of set $I$, leads us to

$$
k_{1}>\frac{1}{\xi_{\min }^{\prime}}\left(\bar{\xi}^{2}(\bar{S})+\xi_{\max }^{\prime} \sum_{i=1}^{2} \rho_{i} \bar{x}_{i} \frac{\bar{\mu}_{i}(\bar{S})-\underline{\mu}_{i}(\underline{S})}{2}\right)
$$

where, for simplicity, $\overline{x_{1}}=x_{1}^{d}$ and $\overline{x_{2}}=x_{2}+\delta$.

Inequalities (b) and (c) are imposed in order to guarantee that $\left(S(t), x_{1}(t)\right.$, $\left.x_{2}(t)\right) \in I$. According to the design of the control on the first step, at the instant of commutation $x_{2}^{d}=x_{2}\left(t_{2}\right), y\left(t_{2}\right)=0$ and $\dot{y_{2}}\left(t_{2}\right)=-x_{2}^{d} \xi\left(S_{m}\right)>0$. Hence, we can obtain estimates on the behaviour of trajectories of $S$ and $x_{2}$ solving analytically the equation $\ddot{y}(t)=-r$ (note that $r>0$ ) for $t \in\left[t_{2}, t_{3}\right]$, where the instant $t_{3}$ corresponds to the first time instant in which $\dot{y}\left(t_{3}\right)=0$.

Consequently, due to properties of the suboptimal control algorithm as shown in Corollary 3.1, we have that:

$$
\begin{aligned}
& |y(t)| \leq y\left(t_{3}\right) \\
& |\dot{y}(t)| \leq y\left(t_{2}\right)
\end{aligned}
$$

and hence, by integration of $\ddot{y}(t)=-r$ in the aforementioned time window, one has that

$$
\dot{y}(t)=\dot{y}\left(t_{2}\right)-r t
$$

and, as $\dot{y}\left(t_{3}\right)=0$ and $\dot{y}\left(t_{2}\right)=-x_{2}^{d} \xi\left(S_{m}\right)$, it is immediate that $t_{3}=\frac{-1}{r} x_{2}^{d} \xi\left(S_{m}\right)$. Integrating once again in the same interval, we have that

$$
y(t)=y\left(t_{2}\right)+\dot{y}\left(t_{2}\right)-\frac{r}{2} t^{2}
$$


which, if evaluated at $t_{3}$, results in inequality (c), i.e. $y\left(t_{3}\right)=\frac{1}{2 r}\left(x_{2}^{d} \xi\left(S_{m}\right)\right)^{2} \leq$ $\delta$.

Finally, inequality (b) is obtained from the second equation in (28), which states that

$$
\left|x_{2}(t) \xi(S(t))\right| \leq x_{2}\left(t_{2}\right) \xi\left(S\left(t_{2}\right)\right)=x_{2}^{d} \xi\left(S_{m}\right)
$$

and, due to monotonicity of $\xi(S)$ for $S>\overline{S_{o}}$, leads us to

$$
\frac{-\left|x_{2}^{d} \xi\left(S_{m}\right)\right|}{x_{2}^{d}+\delta} \leq|\xi(S)| \leq \frac{\left|x_{2}^{d} \xi\left(S_{m}\right)\right|}{x_{2}^{d}-\delta}
$$

which, due to the modulus and the positive nature of the constants $x_{2}^{d}$ and $\delta$, reduces to

$$
|\xi(S)| \leq \frac{\left|x_{2}^{d} \xi\left(S_{m}\right)\right|}{x_{2}^{d}-\delta}
$$

completing the proof.

This last lemma gives us a way to perform a choice of a certain constant gain $k_{1}$ satisfying the aforementioned constraints and to evaluate the domain of attraction $I$. Supposing that there exist the values of $\delta, \underline{S}$ and $\bar{S}$ that verify the constraints in Lemma 4.5, and that the gain $k_{1}$ is selected to satisfy (a), then, we can state the final result of this section as the following theorem:

Theorem 4.6. Consider the dynamics (20) and control law (21). If the constraints described in Lemma 4.5 are satisfied, then the origin of (20) is stabilized in I for all $t>T_{3}$, where $T_{3}>0$ is a finite-time depending on $x_{1}^{d}, x_{2}^{d}$ and $S_{m}$.

Proof. This proof is straightforward by the preliminaries given on the suboptimal control (Subsection 3.2). From (13), we have that (omitting arguments for readability):

$$
T_{3} \leq t_{3}+\alpha p \sqrt{\left|y\left(t_{3}\right)\right|}
$$

where $\alpha\left(S, x_{1}, x_{2}\right)=\max _{I} \frac{b_{\max }\left(x_{2}, S\right) k_{1}+a_{\max }\left(x_{1}, x_{2}, S\right)}{-r}$ and $p$ is defined in Subsection 3.2. Hence, profiting the computations performed in the proof of Lemma 4.5 , we have that

$$
T_{3} \leq \frac{-x_{2}^{d} \xi\left(S_{m}\right)}{r}+\alpha p \sqrt{\left|\frac{1}{2 r}\left(x_{2}^{d} \xi\left(S_{m}\right)\right)^{2}\right|}
$$

as stated.

Remark 4. For the sake of simplicity, the possibility of having time-varying parameters $a$ and $b$ has not been made explicit in the development. However, it can be tackled by the same control architecture in a very natural manner. 
Recalling formula 20), the influence of these time-varying parameters would appear such as

$$
\ddot{y}=x_{2}\left(\xi^{2}(S)-\frac{\partial \xi}{\partial S}\left(D\left(S_{i n}-S\right)-\sum_{i=1}^{2} \rho_{i} x_{i} \mu_{i}(S)\right)-\frac{\partial \xi}{\partial a} \dot{a}-\frac{\partial \xi}{\partial b} \dot{b}\right)
$$

where the two right-most new terms can be seen as a perturbation (especially because $\dot{a}$ and $\dot{b}$ are supposed to be small, given the slow nature of the system) and, taking the uncertainty into account, they can be added to $a_{\max }$. The design of the control law then proceeds as explained in this subsection.

Remark 5. A question that might arise concerns the switching nature of control laws (16), (18) and (21). Although the presented results are theoretical, the slow nature of the studied systems can be advantageous for their implementation (indeed, $D(t)$ is actuated by an electro-mechanical pump and $S_{i n}(t)$ can also be actuated by pumping media with a higher concentration into a lower one). Also, many promising results on the discretization of such control laws (e.g., [26]) might support studies for this realization.

\section{Numerical Example}

In this section, we present a numerical example to verify the usefulness of the developed methodology. Consider model (1) with initial conditions $S(0)=1$ and $x_{1}(0)=x_{2}(0)=1.5$, also considering that the control inputs are constrained such as $D \in[0,2]$ (in $\frac{1}{h}$ ) and $S_{i n}=[0,30]$ (in $\frac{g}{l}$ ). Also, for simplicity, we consider $\rho_{1}=\rho_{2}=1$.

We aim to stabilize $x_{1}$ at $x_{1}^{d}=5$ and $x_{2}$ accordingly, i.e., to stabilize it at the level where it stands when $x_{1}=x_{1}^{d}$ is attained. For simulation purposes only (i.e., the controller does not have such an information), we consider the following parameters sets for the nominal model, as given by Table 1 .

Table 1: Random Parameter Sets

\begin{tabular}{|c|c|c|}
\hline \multicolumn{2}{|c|}{ Table 1: Random Parameter Sets } \\
\begin{tabular}{|c|c|c|}
\hline & {$\left[a_{1}, b_{1}\right]$} & {$\left[a_{2}, b_{2}\right]$} \\
\hline Parameter Set 1 & {$[4.00,20.00]$} & {$[2.00,6.00]$} \\
\hline Parameter Set 2 & {$[3.80,24.00]$} & {$[2.40,7.20]$} \\
\hline Parameter Set 3 & {$[3.60,22.00]$} & {$[2.20,5.40]$} \\
\hline Parameter Set 4 & {$[4.60,23.00]$} & {$[1.60,4.80]$} \\
\hline
\end{tabular}
\end{tabular}

However, for the control synthesis, it is assumed that the parameter uncertainties satisfy the following intervals:

$$
\begin{aligned}
& A_{1}=[3.60,4.60], \quad A_{2}=[1.60,2.40] \\
& B_{1}=[16.00,24.00], \quad B_{2}=[4.80,7.20]
\end{aligned}
$$

and, analysing the impact of these uncertainties in $S_{a, b}$ and $S_{o}$, we can arbitrarily select $S_{m}=7$. Also, in accordance with Lemma 4.5 , the selection of gain $k_{1}$ 
as

$$
k_{1}=0.2\left(\frac{\bar{\xi}^{2}\left(S_{m}\right)}{\xi_{\min }^{\prime}\left(S_{m}\right)}+\underline{\mu}_{1}\left(S_{m}\right)\right)
$$

satisfies all constraints on stabilizability, non-negativeness of $S_{\text {in }}$ and transient behavior of all trajectories will be satisfied for this simulated scenario.

First, let us analyze the whole performance of the control law concerning the parameters given in Table 1. as illustrated in figure 3. As can be seen, both phases successfully stabilizes both species in a finite-time despite of the parametric uncertainties.
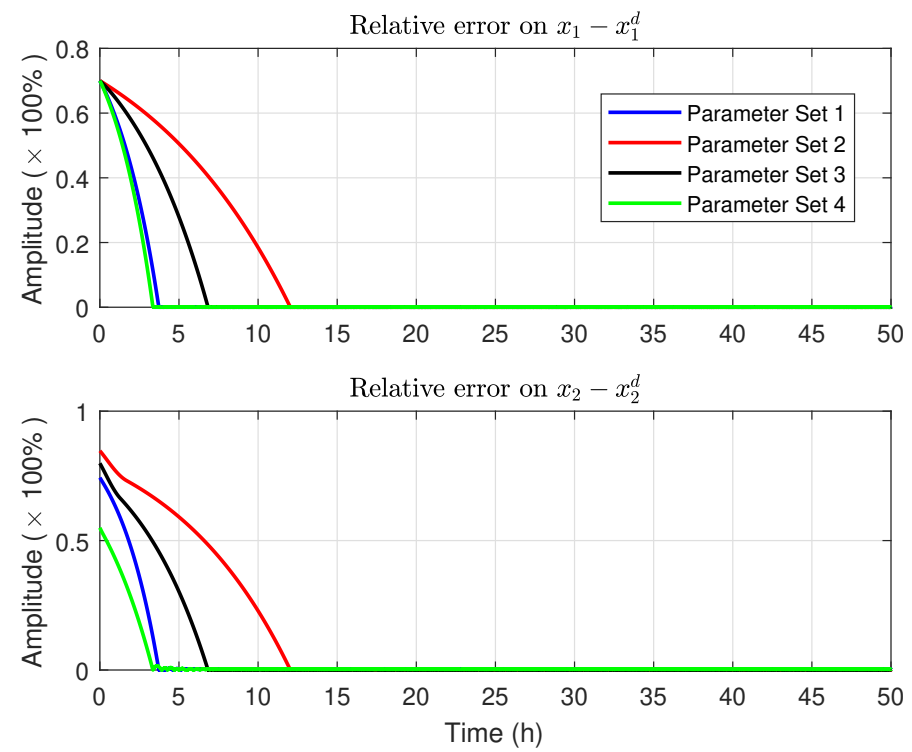

Figure 3: Relative error evolution for both species

Now, for clarity and depth, let us focus only on parameter set 1 . As can be seen in figure 4, the first control phase is activated in $t \in[0,4.7]$. In this interval, one can easily see the stabilization of $S$ at $S_{m}$ and $x_{1}$ at $x_{1}^{d}$. Immediately after this step, the second phase is initiated and successfully stabilizes $x_{2}$ at $x_{2}\left(t_{2}\right)=6.5$.

In figure 5, one can see the time evolution of the control inputs $S_{i n}(t)$ and $D(t)$. Clearly, one can see the sliding-motion of $S_{i n}(t)$ in the first and second phases, where this control input is used to stabilize $S$ and $x_{2}$. Another interesting fact is that, after the activation of the second phase, we can see that $D(t)$ is in its equivalent-control mode, i.e., $D(t)=\mu_{1}(S)$.

Finally, one can see that the control architecture successfully stabilized $S=$ $S_{a, b}$, even though $S_{a, b}$ belongs to an uncertain interval. This fact shows the usefulness of this methodology since it allows a robust solution to the stated stabilization problem. 


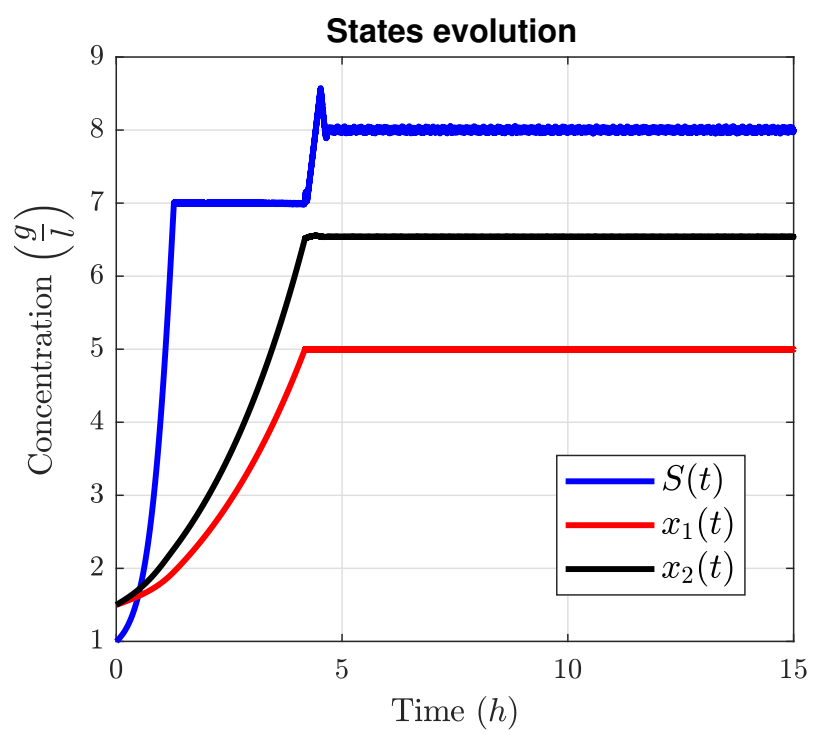

Figure 4: Stabilization at $x_{1}=3$ and $x_{2}=4.13$

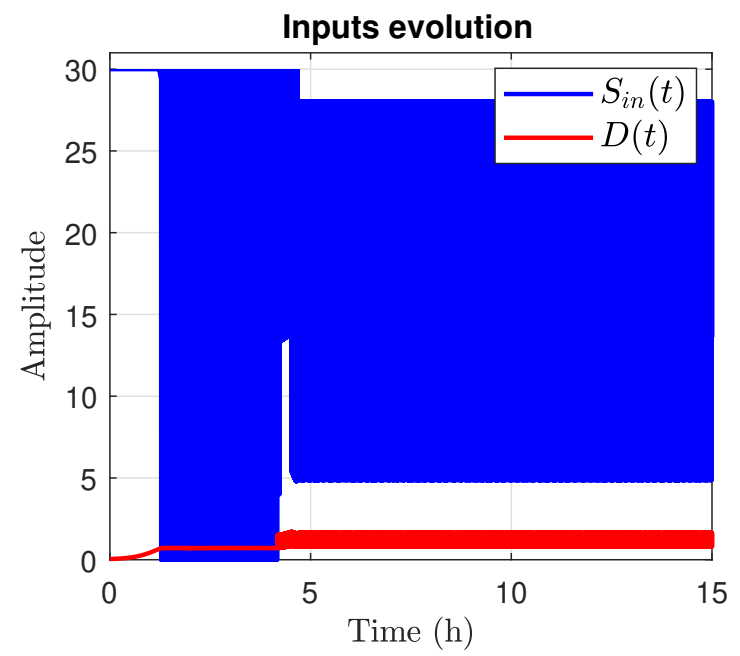

Figure 5: Control inputs $S_{i n}(t)$ and $D(t)$ 


\section{Conclusion}

In this work, we further discussed the problem of stabilization of two species inside a bioreactor, under competition for a single limiting substrate. Considering all parameters on the kinetic rates to be uncertain for the values within a certain given interval, we developed discontinuous control laws that ensure the persistence of all species. These control laws have been designed in two different architectures, which are switched under certain conditions by a supervisor. As it was shown, the concentration of species $x_{1}$ can be globally stabilized at an arbitrary point, while the concentration of species $x_{2}$ can be fixed at a non-zero level.

As a topic of future works, an interesting direction might be pursued by dropping the harsh assumption of full state measurement, implying that the study of observer-based control might be carried out aside from the methodology presented here.

Regarding future practical implementations, as discussed in Remark 5, a study aiming to reduce chattering and the discretization of the sliding mode controllers might be pursued. Also, sensitivity analysis could be interesting to better characterize the effect of the considered uncertainties in the closedloop system, as well as to better verify how large their interval range can reach without compromising the performance of the methodology.

\section{References}

[1] J. Singh, N. Kaushik, S. Biswas, Bioreactors technology \& design analysis, The Scitech Journal (2014) 2347-7318.

[2] P. Leenheer, H. Smith, Feedback control for chemostat models, Journal of Mathematical Biology 46 (2003) 48-70.

[3] F. Mazenc, M. Malisoff, J. Harmand, Further results on stabilization of periodic trajectories for a chemostat with two species, IEEE Transactions on Automatic Control 53 (2008) 66-74.

[4] D. Dochain, G. Bastin, On-line Estimation and Adaptive Control of Bioreactors, Process Measurement and Control, Elsevier, 1990.

[5] G. Robledo, Control of the chemostat: Some Results, Theses, Université Nice Sophia Antipolis (Mar. 2006).

URL https://tel.archives-ouvertes.fr/tel-00069800

[6] C. Kravaris, G. Savoglidis, Tracking the singular arc of a continuous bioreactor using sliding mode control, Journal of the Franklin Institute 349 (2011) 1583-1601.

[7] F. Mairet, O. Bernard, Robustness of Closed-Loop Control to Biodiversity: a Didactic Example, in: 19th IFAC World Congress, Cape Town, South Africa, 2014, pp. 5321-5326. doi:10.3182/20140824-6-ZA-1003.02193. URL https://hal .inria.fr/hal-01094693 
[8] J. Gouzé, G. Robledo, Robust control for an uncertain chemostat model, International Journal of Robust and Nonlinear Control 16 (3) (2006) 133155.

[9] D. Dochain, Automatic Control of Bioprocesses, Control systems, Robotics and Manufacturing Series, ISTE Ltd., 2008.

[10] H. L. Smith, P. Waltman, The theory of the Chemostat, Cambridge University Press, 1995.

[11] H. C. Bernstein, S. D. Paulson, R. P. Carlson, Synthetic escherichia coli consortia engineered for syntrophy demonstrate enhanced biomass productivity, Journal of Biotechnology 157 (1) (2012) 159 - 166.

[12] W. Perruquetti, J. Barbot (Eds.), Sliding Mode Control in Engineering, Control Engineering Series, Marcel Dekker, 2002.

[13] A. Rapaport, J. Harmand, Robust regulation of a class of partially observer nonlinear continuous bioreactors, Journal of Process Control 12 (2002) 291302.

[14] Z. Duan, C. Kravaris, Robust stabilization of a two-stage continuous anaerobic bioreactor system, AIChE Journal 64 (4) (2018) 1295-1304. URL https://aiche.onlinelibrary.wiley.com/doi/abs/10.1002/ aic. 16033

[15] J. A. Spriet, Modelling of the growth of microorganisms a critical appraisal, Environmental Systems, Analysis and Management (1982) 451-456.

[16] A. Reis de Souza, D. Efimov, A. Polyakov, J. Gouzé, Robust control of a competitive environment in the chemostat using discontinuous control laws, 58th Conference on Decision and Control (2019).

[17] H. L. Smith, Competitive coexistence in an oscillating chemostat, SIAM J. Appl. Math. 40 (3) (1981) 498-522.

[18] N. S. Rao, E. O. Roxin, Controlled growth of competing species, SIAM J. Appl. Math. 50 (3) (1990) 853-864.

[19] S. B. Hsu, S. Hubbell, P. Waltman, A mathematical theory for singlenutrient competition in continuous cultures of micro-organisms, SIAM J. Appl. Math. 32 (2) (1977) 366-383.

[20] Y. Shtessel, C. Edwards, L. Fridman, A. Levant, Sliding Mode Control and Observation, Control Engineering, Birkhauser, 2010.

[21] S. Emelyanov, S. Korovin, L. Levantovskii, High-order sliding modes in binary control systems, Soviet Physics Doklady 31 (1986).

[22] G. Bartolini, A. Ferrara, E. Usai, Output tracking control of uncertain non linear second order systems, Automatica 33 (1997) 2203-2212. 
[23] A. Levant, Quasi-continuous high-order sliding-mode controllers, IEEE Transactions on Automatic Control 50 (11) (2005) 1812-1816.

[24] A. Polyakov, A. Poznyak, Unified lyapunov function for a finite-time stability analysis of relay second order sliding mode control systems, IMA Journal of Mathematical Control and Information (2012) 529-550.

[25] V. I. Utkin, Sliding Modes in Control and Optimization, Communications and Control Engineering, Springer, 1992.

[26] A. Polyakov, D. Efimov, B. Brogliato, Consistent Discretization of Finitetime and Fixed-time Stable Systems, SIAM Journal on Control and Optimization 57 (1) (2019) 78-103. doi:10.1137/18M1197345.

URL https://hal.inria.fr/hal-01838712 